Proceedings

\title{
A Technology Platform For the Sustainable Recovery and Advanced Use of Nanostructured Cellulose from Agri-Food Residues (PANACEA Project) ${ }^{\dagger}$
}

\author{
Francesco Donsì 1,*, Simonetta Bartolucci ${ }^{2}$, Paolo Bettotti ${ }^{3}$, Federico Carosio ${ }^{4}$, Patrizia Contursi ${ }^{2}$, \\ Gennaro Gentile ${ }^{5}$, Marina Scarpa ${ }^{3}$ and Giorgia Spigno ${ }^{6}$ \\ 1 Università degli Studi di Salerno, Italy \\ 2 Università degli Studi di Napoli Federico II, Italy \\ * Correspondence: fdonsi@unisa.it; Tel.: +39-089-96-4135 \\ † Presented at the First International Conference on “Green" Polymer Materials 2020, 5-25 November 2020; \\ Available online: https://cgpm2020.sciforum.net/.
}

Published: 4 November 2020

\begin{abstract}
The European food sector generates about 250 million ton/yr of by-products and waste, of which around $10 \%$ from fruit and vegetable processing, with a heavy environmental burden. The agri-food residues (AFRs) contain a significant fraction of cellulose and bioactive compounds, which, if recovered, are high added-value material components. The reduction of cellulose down to nano-sized crystalline structures (nanocellulose, NC) provides versatile building blocks, which selfassemble into new materials with superior performances. The PANACEA project, within the frame of PRIN 2017 call supported by the Italian Ministry of University and Research, proposes an approach based on the recovery of cellulose and bioactive compounds from AFRs, with high yield, at various degrees of hierarchical organization, by cascading different physical and chemical processes of increasing complexity, including physical processes and microbial digestion to obtain micro-sized cellulose structures while preserving their bioactivity. Chemical and enzymatic processes are used to isolate, purify and functionalize NC at different levels of hierarchical organization, and to design advanced functional materials such as food ingredients, edible coatings, functional colloids, biocides, and flame retardants.
\end{abstract}

Keywords: process engineering; food additives and residues; integrated and sustainable management of resources and residues; synthesis of biomaterials; biomaterials; cellulose

\section{Introduction}

The European food sector generates about 250 million ton/yr of by-products and waste, of which around $10 \%$ from fruit and vegetable processing [1,2]. Such residues are mainly used as animal feed, fertilizers, or dietary fiber. However, a large part is discarded, with a heavy environmental burden $[2,3]$. In line with the Horizon 2020 objectives, the following challenges to the sustainable valorization of agri-food residue (AFRs) can be identified:

- Simple, sustainable, and environmentally friendly processes;

- Cost-effective process cascades to valorize added-value compounds;

- Development of new functionalities and application markets.

The AFRs on average consist of a significant fraction of non-starch polysaccharides (35-60\% dietary fiber), including pectins (2-13\%), cellulose (7-44\%), hemicelluloses (4-33\%), lignins (15-69\%), and gums, depending on the source $[4,5]$.

In particular, cellulose is one of the building blocks of plant life, and the most abundant organic compound on earth [2]. Owing to its hierarchical structure, and the organization of its elementary 
nanofibrillar components in natural fibers, cellulose confers unique properties to different species of plants, and high mechanical strength and high strength-to-weight ratio while allowing for significant flexibility [6].

Cellulose can be broken down to produce nano-sized crystalline structures collectively referred to as nanocellulose (NC). NC exhibits advanced functionalities, which may contribute to add value to existing materials or develop new ones, by conferring superior performances and extensive applications, i.e. in polymer, textile, pharmaceutical, biomedical, and food industries [7].

Despite wood NC is generally considered a green material, its production process is environmentally unfriendly and its large scale utilization would contribute to deforestation. Therefore, more sustainable sources such as AFRs, and greener production routes are desired. Furthermore, AFRs still contain precious nutrients that can be recovered by designing specific product transformations.

The PANACEA project (A technology platform for the sustainable recovery and advanced use of nanostructured cellulose from agri-food residues) aims to develop a technology platform able to process AFRs for the recovery of cellulose and high value-added compounds to be used as building blocks to develop more sustainable advanced materials.

The specific objectives of the project are:

O.1 Production of intermediate cellulose materials with bioactive molecules from AFRs by proprietary high-pressure technology.

O.2 Microbiome selection to degrade intermediate cellulose products.

O.3 Development of processes to isolate, purify, functionalize, and characterize NC at different levels of hierarchical organization.

O.4 Design of advanced functional materials such as food ingredients, edible coatings, functional colloids, biocides, and flame retardants.

The proposed approach is to develop a strategy to recover cellulose from AFR at different degrees of hierarchical organization, by cascading different physical, chemical, and enzymatic processes of increasing complexity, as sketched in Figure 1. The initial treatments (mainly physical processes) are the simpler ones and can be carried out at the site of origination of the AFRs, while the following more complex ones require centralized and specialized sites.

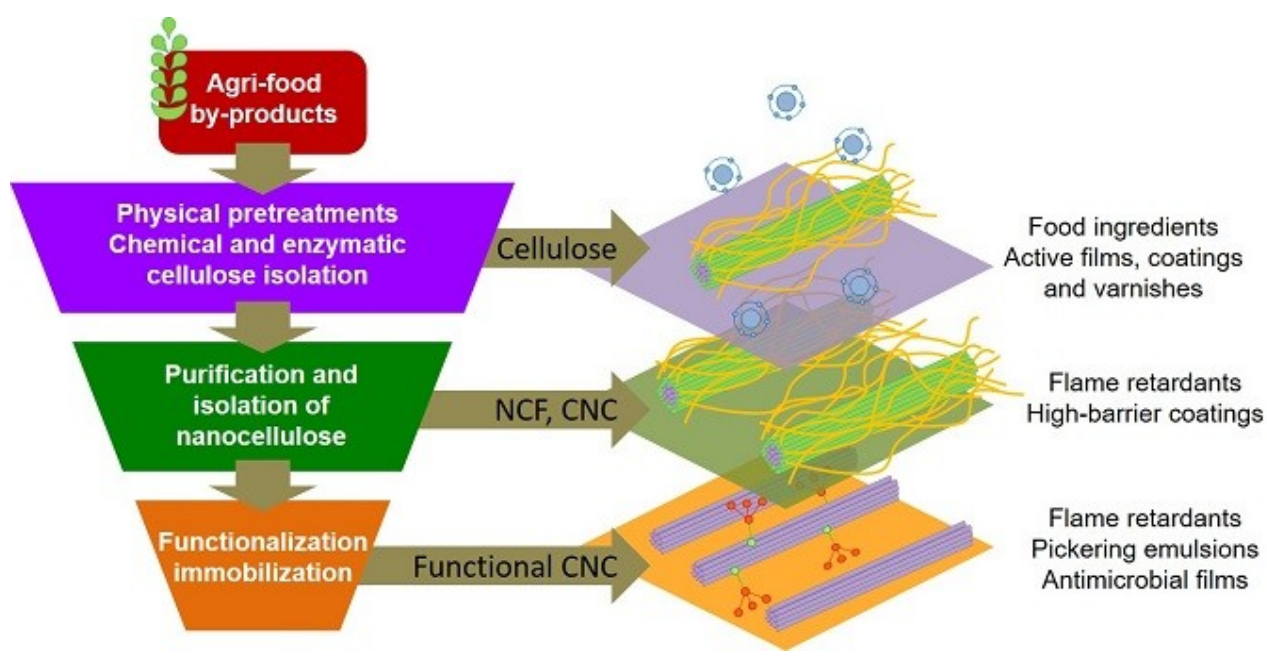

Figure 1. Sketch of the envisioned recovery of different hierarchical NC structures from AFRs, for advanced applications.

\section{State of the Art}

NC can be derived from a variety of sources. Wood sources contain relevant amounts of hemicellulose and lignin, hence requiring intense mechanical or chemical treatments. Therefore, lowlignin raw materials, and non-wood sources are receiving increasing attention [8]. Cellulose is present in almost all the AFRs, even though in different amounts and with different properties in terms of 
accessibility and morphology. The major cellulosic wastes from agro-food industries are damaged fruits, vegetables, grains, and post-processing residues (peels, seeds, leaves, husks, pomaces, etc.) [9]. Almost any cellulosic material can be considered as a potential source of cellulose nanocrystals/fibers, differing in the availability of the material, percentage of cellulose, the yield of recovery, size of the isolated NC structures.

NC can be divided into two main categories: (i) cellulose nanocrystals (CNC) or cellulose whiskers and (ii) cellulose nanofibrils (CNF) [8]. CNC are compact, rod-like, and almost crystalline in nature, while CNF are entangled networks of fibers with some kinks and amorphous regions. In both cases, the polymer chains are inter-connected by a strong hydrogen bond network and Van der Waals interactions [10].

Several production routes of NC have been reported and extensively reviewed $[8,11,12]$. The first step usually consists of alkali, bleaching, or cooking treatments of the raw material to obtain pretreated cellulose, followed by a mechanical defibrillation process. Dry milling leads to fiber shredding, rather than elementary fibril delamination (poor mechanical properties) and, therefore, wet milling in an aqueous medium is preferred to loosen the inter-fibrillar hydrogen bonding.

Biological (enzymatic hydrolysis) and chemical pretreatments (carboxylation, sulfonation, carboxymethylation, quaternization) might integrate or replace mechanical disintegration, reduce energy costs, but strongly influence NC properties.

The currently available techniques generally yield a wide size distribution of produced CNF with some non-fibrillated residual fibers. Thus, it is very important to develop efficient fractionation tools, possibly integrated into the mechanical process for retrieving the undesired large fragments, for further disintegration.

Several remarkable NC properties (mechanical, chemical, and optical) have been exploited to develop NC-based advanced materials. For example, NC is used in biomaterials, energy storage, water treatment, composite materials [13]. In foods, due to its rheological behavior and water absorption, NC can find use as (i) stabilizing or emulsifying agent, (ii) functional food ingredient, or (iii) food packages [14].

PANACEA project aims at significantly advancing the knowledge in the field of cellulose recovery from AFRs by investigating the tailored valorization of different AFRs, ranging from total extraction (cellulose and bioactive molecules) for food ingredients, to the production of CNF and $\mathrm{CNC}$ with defined functionalities, as well as contributing to developing greener and more sustainable processes, while preserving raw materials functionalities. The cascading approach consists of exploiting purely physical processes in the initial treatment steps (eventually combined with mild enzymatic reactions), followed by chemical functionalization, to transform CNF and CNC into added-value fine chemical building blocks for advanced materials. PANACEA project has also the ambition to contribute to opening new routes (from extraction to application) for the valorization of AFRs into sustainable and high performing materials, characterized by improved gas barrier properties or flame retardant characteristics, as well as to developing advanced materials for the food industry (edible coatings, ingredients, and packaging), foams and textiles with biocide, gas-barrier, and fire-retardant properties.

\section{Methodology}

PANACEA is organized in 4 work packages (WP), shown in Figure 2, with cascading processes for recovering cellulose from different AFRs (WP1) and transforming them into functional nanostructures at different levels of hierarchical organization (WP2), which are characterized (WP3) and exploited in different materials or applications (WP4). Dissemination activities (WP5) involve the foreground generated in all the research work packages. 


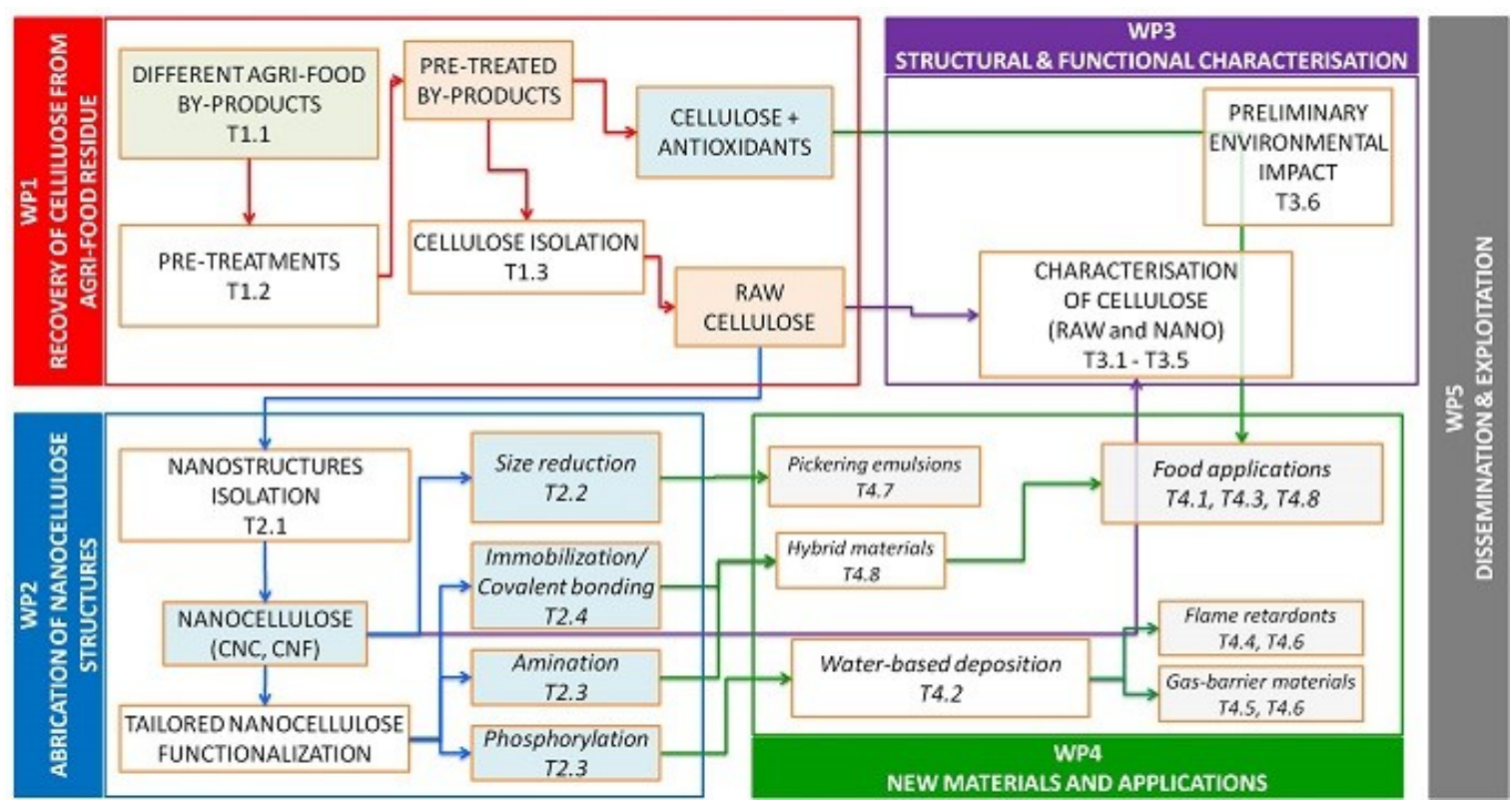

Figure 2. Schematics of the project methodology expressed as networked work packages.

\subsection{WP1-Cellulose from Agri-Food Residues}

WP1 is addressed to developing a sustainable strategy for the integrated valorization of AFRs through the recovery of cellulose and other added-value compounds. It is articulated in the following tasks:

T1.1: Ad hoc pre-treatment of different AFRs, selected also based on their availability during the year (Tomato peels and wheat straws, available in summer; coffee ground residues and rice bran, available all year round; grape marcs, available in summer-fall; orange peels, available in winter), chemical characterization and assessment of extractability of cellulose by conventional technologies.

T1.2: Application of proprietary high-pressure homogenization (HPH) technology to micronize the residues in water and completely disintegrate the vegetable cells, unlocking all the intracellular and structural materials. Fractionation of the micronized residues is integrated into the HPH, to recover insoluble fibers, enriched with bioactives, for food applications [15], further processing (WP1 and WP2), or film-forming materials.

T1.3: Separation of hemicelluloses - lignin - cellulose, which is carried out via conventional chemical fractionation based on double acid/basic step hydrolysis or by a high-pressure/high-temperature autohydrolysis step. Combination of the HPH treatment with chemical or enzymatic pretreatments or post-treatments enables to specifically target the recovery of cellulose and NC (preparatory to WP2).

\subsection{WP2-Fabrication of NC Structures}

WP2 is addressed to the fabrication of NC structures (CNC, CNF) with tunable size, crystallinity, and surface properties, to bridge the gap between the production of cellulose and cellulose hybrids and the final applications. It is articulated in the following tasks:

T2.1: Size-reduction of NC and variation of the ratio between crystalline and amorphous regions, via harsh chemical oxidation or mild enzymatic lysis, to produce CNF or CNC. The enzymemediated biological degradation is applied to fringe the crystallites, to provide the site for the easier introduction of functional groups (T2.3).

T2.2: Study of wet milling processes, such as HPH, ball milling, or ultrasounds, to i) improve the water dispersion of NC; ii) tailor the average length and diameter of CNF; iii) activate the CNF surface in view of further chemical modifications; iv) partially disintegrate the amorphous 
cellulose chains while maintaining contact with the crystalline body, for the formation of protruding soft biopolymer chains.

T2.3: Reaction protocols for NC functionalization. CNC and CNF are supramolecular structures only marginally stable in solution. Amination enables the formation of hybrid materials with greatly different mechanical properties, or the production of polyampholytes, which undergo optimized interaction with biological systems [16]. Phosphorylation might contribute to developing flame retarding properties.

T2.4: Functionalization with limonene, through (i) physical immobilization in NC hydrogel microbeads to protect limonene from oxidation, increase the water dispersion and enable its controlled release, or (ii) covalent bonding to CNCs for fabricating building-blocks, eventually hybridized with inorganic fillers (silicates or graphene oxide), to develop antimicrobial materials with controlled release properties.

\subsection{WP3-Structural and Functional Characterization}

WP3 focuses on NC characterization in terms of yields, purity, physicochemical, structural, and functional properties, through the following tasks:

T3.1: A multi-technique approach is applied to the products obtained from WP1 and WP2, based on infrared spectroscopy (FTIR), solid-state NMR spectroscopy, scanning electron microscopy (SEM), transmission electron microscopy (TEM), X-ray diffraction (XRD), thermal and thermogravimetric analysis. The size of cellulose agglomerates dispersed in water and the stability of the dispersions are evaluated by dynamic light scattering and the specific surface area through gas sorption techniques. The cellulose purity (content of lignin, cellulose, and hemicellulose), and the degree of fibrillation are also determined.

T3.2: The influence of the wet milling processes on the bio-accessibility (through the simulated digestive process) of bioactive compounds and fibers is evaluated

T3.3: The filming capacity of the micronized residues or the separated fractions is assayed through the addition of suitable additives (such as food-grade plastifying agents) to obtain edible coatings for innovative food applications, such as encapsulation of fruit purees/juices.

T3.4: Evaluation of the gas-barrier properties of cellulose and NC recovered in WP1 and WP2.

T3.5: Determination of rheological properties of cellulose and NC, in terms of shear stress and viscosity of NC suspensions at equilibrium.

T3.6: The cellulose yields, purity of NC, and associated energy, water, and reagents consumptions are determined to contribute to process sustainability assessment.

Through an iterative process, the results obtained in WP3 allow the optimization of the processes described in WP1 and WP2.

\subsection{WP4-New Materials and Applications}

WP4 is addressed to the exploitation of NC-based colloids for food applications, as well as pursuing water-based deposition processes for gas barrier properties or flame retardant characteristics, and biobased nanocomposites with advanced properties. It is articulated as follows:

T4.1: Development of edible coatings, antimicrobial varnishes, and oil structuring materials, using the cellulose-based output materials from WP1.

T4.2: Water-based deposition process for NC-based colloids (from WP2) on selected substrates (biobased films, flexible foams, and fabrics), as schematized in Figure 3.

T4.3 Packaging films treated with gas-barrier coatings are tested evaluating oxygen and water vapor permeability at different temperatures and relative humidity values.

T4.4 Gas-barrier coatings are tailored to limit the coating swelling in a humid environment, to prevent the loss of gas barrier properties. The development of cross-linkable coatings will also improve the washing and deformation cycle resistance properties for long term applications for textile and flexible foams, respectively. 


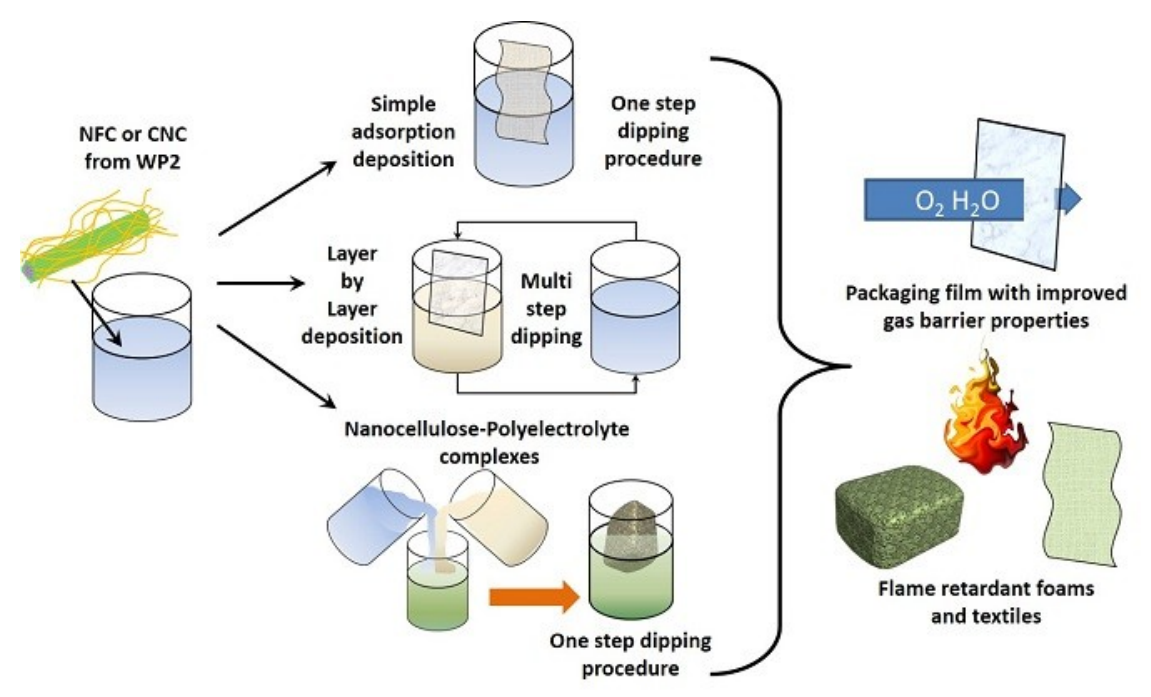

Figure 3. Schematics of NC water-based depositions processes on different materials.

T4.5 Fabrics and foams treated with flame retardant coatings are thoroughly characterized for flame retardancy. Flammability and cone calorimetry tests permit to check the efficiency of the deposited coatings in a realistic fire scenario when free flames can propagate.

T4.6 Realization of advanced nanocomposite films, based on polylactic acid (PLA), and poly(ecaprolactone) (PCL), and foams, based on biopolyurethanes (bioPU) containing NC as nanofillers and treated with high-gas barrier coatings and flame retardant coatings. These materials will also be tested as flame retardants.

T4.7 CNC as a stabilizer to form Pickering emulsions. Emulsions properties and stability is studied vs. solvent parameters, in strong collaboration with the characterization techniques of WP3.

T4.8 Antimicrobial films and coatings are developed exploiting the natural antimicrobial properties of total extracts from the residues (WP1) and immobilized limonene (WP2).

\section{Discussion}

Several elements of novelty are involved in this project. First of all, the application of proprietary technology (modified HPH system) represents an original approach to develop a simpler and greener recovery of cellulose, by avoiding/reducing chemical pretreatments and using only coarsely milled materials. The aqueous AFR suspensions obtained by HPH are made of finely milled cellular debris, where cellulose is partly defibrillated and activated. This technology has already been tested on tomato peels, spent coffee grounds, sesame seeds, and vegetable tissue [16-18]. The full exploitation of micronized by-products in final products, such as edible coatings and films, or oil structuring systems, investigated in WP4, by combining partly activated cellulose with other bioactives naturally contained in the residues [15], also represents an original approach.

The use of high-pressure/high-temperature autohydrolysis to increase CNC yield also represents a novel approach, especially in combination with the HPH treatment, as it is expected to greatly influence the separation of cellulose from the other components. Finally, the combination of intensive wet milling with enzymatic treatments has not been studied previously.

The tailored use of enzymes to achieve high yields of NC with higher selectivity, lower energy costs, and milder operating conditions than chemical processes [17], as well as the use of cellobiome of bacterial origin to obtain NC of variable size and relative content of amorphous and crystalline domains [18], represent other elements of novelty in cellulose recovery from AFRs.

Advancement of the knowledge can also be expected from exploiting physical treatments, such as ball milling or $\mathrm{HPH}$, to induce the formation of highly amorphous nanometric cellulose particles as well as CNF with an average diameter of about $100 \mathrm{~nm}$, also in combination with mild hydrolysis conditions. The obtained NC will be subjected to chemical modification, for example, through the 
development of novel thiol-ene reactions to covalently attach limonene to $\mathrm{CNC}$ and $\mathrm{CNF}$, for improving its potential application in different fields. For example:

1. The suspensions, where intracellular compounds are fully unlocked, and cellulose is partly defibrillated (WP1), will be used for oil structuring, or active coatings containing natural antioxidant compounds and proteins, as preliminary demonstrated in pioneering works [15].

2. The CNC compact nanostructures, thanks to the hydroxyls groups exposed at the surface, provide the interface for the self-assembly of the individual nanostructures into macroscopic phases such as hydro- or aerogels, foams, and films, or chemical modification, opening new opportunities for innovative applications.

3. Replacement of traditional flame-retarding polymers, based on halogenated additives, posing severe environmental and health concerns, with approaches employing NC as building block for gas barrier and flame retardancy coatings,

4. Enhancement of biopolymers gas barrier properties thanks to the use of NC in either multilayered high barrier thin coatings or as biobased filler.

5. Realization of novel biobased or biodegradable polymer nanocomposite films (PLA, PCL) with advanced gas-barrier properties and biobased nanocomposite foams (bioPU) with advanced flame retardant properties, obtained by the effective embedding of properly functionalized NC within the polymer matrices and the application of functional NC-based coatings.

6. Nanoparticles-stabilized emulsions (Pickering emulsions) form more stable suspensions than soluble surfactants. CNC is a promising, yet underused, material to develop such systems because of the large surface energy and the possibility to control the surface chemistry. Emulsion droplet size depends on the size of NC particles, which is hence the critical parameter to be controlled in WP2.

7. Terpenes (i.e. limonene) are a versatile chemical platform for new synthetic building blocks. The combination of bioactivity and reactivity of limonene with the mechanical and structural properties of $\mathrm{CNC}$ is expected to add functionalities, in particular as natural biocides and pestcontrol agents, and flame-retardant materials, which have not been explored before.

\section{Conclusions}

The PANACEA project, within the frame of PRIN 2017 call supported by the Italian Ministry of University and Research, proposes an approach based on the recovery of cellulose and bioactive compounds from AFRs, with high yield, at various degrees of hierarchical organization, by cascading different physical and chemical processes of increasing complexity. More specifically, physical processes and microbial digestion are exploited to obtain micro-sized cellulose structures while preserving their bioactivity. Chemical and enzymatic processes are used to isolate, purify and functionalize NC at different levels of hierarchical organization, and to design advanced functional materials such as food ingredients, edible coatings, functional colloids, biocides, and flame retardants.

Author Contributions: All authors conceived and designed the experiments and wrote the paper.

Acknowledgments: This work was supported by the Italian Ministry of University (MUR) call PRIN 2017 with the project 2017LEPH3M "PANACEA: A technology PlAtform for the sustainable recovery and advanced use of NAnostructured CEllulose from Agro-food residues." The project provided funds for covering also the costs to publish in open access.

Conflicts of Interest: The authors declare no conflict of interest.

\section{References}

1. Fava, F.; Totaro, G.; Diels, L.; Reis, M.; Duarte, J.; Carioca, O.B.; Poggi-Varaldo, H.M.; Ferreira, B.S. Biowaste biorefinery in Europe: Opportunities and research \& development needs. N. Biotechnol. 2015, 32, 100-108.

2. Szymanska-Chargot, M.; Chylinska, M.; Gdula, K.; Koziol, A.; Zdunek, A. Isolation and characterization of cellulose from different fruit and vegetable pomaces. Polymers. 2017, 9, 495. 
3. Nawirska, A.; Uklanska, C. Waste products from fruit and vegetable processing as potential sources for food enrichment in dietary fibre. Acta Sci. Pol. Technol. Aliment. 2008, 7, 35-42.

4. Dhillon, G.S.; Kaur, S.; Brar, S.K. Perspective of apple processing wastes as low-cost substrates for bioproduction of high value products: A review. Renew. Sustain. Energy Rev. 2013, 27, 789-805.

5. Nawirska, A.; Kwaśniewska, M. Dietary fibre fractions from fruit and vegetable processing waste. Food Chem. 2005, 91, 221-225.

6. Dufresne, A. Nanocellulose: A new ageless bionanomaterial. Mater. Today 2013, 16, 220-227.

7. Chen, H.; Seiber, J.N.; Hotze, M. ACS select on nanotechnology in food and agriculture: A perspective on implications and applications. J. Agric. Food Chem. 2014, 62, 1209-1212.

8. Nechyporchuk, O.; Belgacem, M.N.; Bras, J. Production of cellulose nanofibrils: A review of recent advances. Ind. Crops Prod. 2016, 93, 2-25.

9. Das, H.; Singh, S.K. Useful Byproducts from Cellulosic Wastes of Agriculture and Food Industry - A Critical Appraisal. Crit. Rev. Food Sci. Nutr. 2004, 44, 77-89.

10. Shen, T.; Gnanakaran, S. The stability of cellulose: A statistical perspective from a coarse-grained model of hydrogen-bond networks. Biophys. J. 2009, 96, 3032-3040.

11. Klemm, D.; Kramer, F.; Moritz, S.; Lindström, T.; Ankerfors, M.; Gray, D.; Dorris, A. Nanocelluloses: A new family of nature-based materials. Angew. Chemie - Int. Ed. 2011, 50, 5438-5466.

12. Afrin, S.; Karim, Z. Isolation and Surface Modification of Nanocellulose: Necessity of Enzymes over Chemicals. ChemBioEng Rev. 2017, 4, 289-303.

13. Mondal, S. Preparation, properties and applications of nanocellulosic materials. Carbohydr. Polym. 2017, 163, 301-316.

14. Gómez H., C.; Serpa, A.; Velásquez-Cock, J.; Gañán, P.; Castro, C.; Vélez, L.; Zuluaga, R. Vegetable nanocellulose in food science: A review. Food Hydrocoll. 2016, 57, 178-186.

15. Ferrari, G.; Mustafa, W.; Donsì, F. Use of agri-food residues for oil structuring and functionalization. Chem. Eng. Trans. 2017, 57, 1831-1836.

16. Jurić, S.; Ferrari, G.; Velikov, K.P.; Donsì, F. High-pressure homogenization treatment to recover bioactive compounds from tomato peels. J. Food Eng. 2019, 262, 170-180.

17. Donsì, F.; Velikov, K.P. Mechanical cell disruption of mustard bran suspensions for improved dispersion properties and protein release. Food Funct. 2020, 11, 6273-6284.

18. Gali, L.; Bedjou, F.; Velikov, K.P.; Ferrari, G.; Donsì, F. High-pressure homogenization-assisted extraction of bioactive compounds from Ruta chalepensis. J. Food Meas. Charact. 2020, 14, 2800-2809.

Publisher's Note: MDPI stays neutral with regard to jurisdictional claims in published maps and institutional affiliations.

(C) 2020 by the authors. Submitted for possible open access publication under the terms and conditions of the Creative Commons Attribution (CC BY) license (http://creativecommons.org/licenses/by/4.0/). 